\title{
Anglais de spécialité et plurilinguisme
}

\section{Étienne Guyon et Anne Guyon}

\section{OpenEdition}

\section{Journals}

Édition électronique

URL : http://journals.openedition.org/asp/3394

DOI : 10.4000/asp.3394

ISSN : 2108-6354

\section{Éditeur}

Groupe d'étude et de recherche en anglais de spécialité

\section{Édition imprimée}

Date de publication : 1 décembre 1996

Pagination : 1-12

ISSN : 1246-8185

\section{Référence électronique}

Étienne Guyon et Anne Guyon, « Anglais de spécialité et plurilinguisme », ASp [En ligne], 11-14| 1996, mis en ligne le 01 avril 2013, consulté le 01 mai 2019. URL : http://journals.openedition.org/asp/3394 ; DOI : 10.4000/asp.3394

Ce document a été généré automatiquement le 1 mai 2019.

Tous droits réservés 


\title{
Anglais de spécialité et plurilinguisme
}

\author{
Étienne Guyon et Anne Guyon
}

1 L'invitation faite au physicien Étienne Guyon de parler à l'ouverture de ce XVII colloque du GERAS ne pouvait que l'inciter à se présenter lui-même comme sujet d'expérience de sa propre pratique de l'anglais de spécialité. Compte tenu de l'importance et de la spécificité d'une analyse plus proprement linguistique, cette communication a constitué l'occasion de collaborer avec une jeune linguiste, Anne Guyon. Nous avons ensemble ainsi prolongé cette introspection par quelques généralisations sur les avantages et les limites de l'usage de l'anglais véhiculaire pour les échanges scientifiques internationaux. Nous avons aussi envisagé la perspective du plurilinguisme comme une autre solution d'optimisation de l'expression et de la communication spécialisée. Bien que cet article ait été écrit et préparé à deux, nous avons gardé le style du témoignage personnel à une voix qui était celui de la communication orale faite à Aix-en-Provence.

\section{Vertus de mon « bilinguisme»}

2 J'ai été un élève médiocre en anglais tout au long de ma scolarité, mais j'ai passé par la suite quatre années de ma vie professionnelle comme enseignant et chercheur aux ÉtatsUnis. Ceci a fait de moi ce qu'on appelle un «scientifique bilingue». Mon C.V. contient d'ailleurs plus de $80 \%$ d'articles originaux en anglais. Ceux en français sont le plus souvent des notes données aux comptes rendus de l'Académie des sciences, des articles de revue et des livres de synthèse et d'enseignement. On note déjà une certaine spécialisation de chacune des deux langues d'expression: l'anglais pour annoncer des résultats, le français pour les stabiliser.

3 Mon bilinguisme s'entend comme la maîtrise, à côté de celle de ma langue maternelle, d'une langue codée efficace. Elle permet, plus simplement même que dans ma langue maternelle, de rédiger les articles en accolant des formules lues dans d'autres articles de la même discipline et réutilisées telles quelles par suite d'une assimilation le plus souvent inconsciente. La taille de ces formes lexicales et syntaxiques figées est souvent assez importante. Les extraits originaux donnent lieu à réarrangement de ces éléments formels 
et internationalement validés. On peut, naturellement, caricaturer cette pratique d'expression en la comparant à un exercice de «couper/coller " plus ou moins habile et plus ou moins pertinent. J'avancerai que ces formes linguistiques s'apparentent aux autres outils d'expression internationaux et formels de la science que sont les tableaux, les figures, les formules mathématiques et, même, les références bibliographiques.

Un tel langage est d'une grande commodité, en raison même de sa relative pauvreté, de l'absence de références culturelles plus personnelles et profondes. Ceci semble même une condition nécessaire pour les contacts variés et différenciés que nécessitent les échanges écrits ou oraux au sein d'une communauté internationale hétérogène en dehors de sa pratique commune de la science. Il est en effet, plus facile de disposer d'un code minimal, conforme à l'objet réputé universel (la Science) qu'il manipule que de s'encombrer d'une langue trop riche en connotations culturelles lesquelles sont évidemment moins universellement partagées.

\section{Limites de mon « bilinguisme »}

5 Ce bilinguisme dont je ne voudrais pas m'enorgueillir trop vite est bien particulier. Mes capacités en anglais présentent un certain nombre de faiblesses, tant à l'écrit qu'à l'oral, que je voudrais présenter.

Écrit

6 Dans le registre de l'écrit, un anglophone ou américanophone remarquera très vite quelques maladresses dans mon style, ne serait-ce qu'en raison du mélange d'expressions ou d'orthographes anglaise et américaine (les terminaisons or, our ; er, re; se, ze !), ou de la césure en fin de ligne, qui n'en sont que les moins graves. De telles maladresses témoignent de difficultés que les auteurs non anglophones en général essayent néanmoins de maîtriser par des efforts et un temps de rédaction supérieur. En outre, audelà du coût pour le rédacteur lui-même, cela a des conséquences sur la lisibilité de sa prose. Mon expérience de referee de revues internationales en langue anglaise est que la lecture que je dois faire d'articles d'auteurs non anglophones est rendue bien plus difficile lorsque les erreurs stylistiques viennent constamment brouiller le sens, ou au minimum en gêner la lecture. Les referees s'adonnent souvent à la tâche ingrate de corriger des fautes de ce niveau-là pour permettre qu'un article devienne accessible.

7 Je prends pleinement conscience de l'obstacle que constitue la langue, même dans sa version simplifiée, lorsque je partage l'écriture d'un article avec un anglophone. L'interaction avec d'authentiques locuteurs procure alors un tout autre plaisir qui me fait quitter les mécanismes de "couper/coller» dont je parlais précédemment. J'ai même pratiqué une fois l'exercice d'écrire sous forme de poème un travail fait avec un Anglais et un Américain ${ }^{1}$. Pour ce faire, nous avons dû employer un anglais plus classique, au lexique plus riche et le manipuler de façon beaucoup plus subtile qu'habituellement dans un article scientifique et j'ai pu faire pleinement l'expérience de mon inaptitude à versifier en anglais. Ce qui donne à penser que ce texte aura posé quelques problèmes de lecture aux physiciens non anglophones.

Oral

Dans le registre de l'oral, on peut distinguer les communications faites dans un colloque des échanges interpersonnels qui sont souvent les moments les plus importants dans un congrès. Lorsque je suis à la tribune d'un colloque et que je communique, je n'ai aucune 
gêne à m'exprimer ni n'éprouve de difficultés particulières pour répondre aux questions² ${ }^{2}$. De même, je n'ai pas grande difficulté à recevoir le message d'un orateur anglophone ou non ayant structuré et élaboré son intervention dans le style propre au genre, en l'accompagnant de documents écrits. En revanche, je me trouve désavantagé par rapport aux anglophones dans un débat. La seconde de décalage dans la compréhension d'une intervention qui utilise souvent alors une forme plus spontanée, moins codée que les communications orales elles-mêmes, est un handicap dans cette phase où s'élaborent des savoirs nouveaux, où sont remises en cause des vérités tenues pour sûres. On aborde là, un aspect qui va bien au-delà des questions techniques de maitrise linguistique vue comme la pratique d'une combinaison de règles grammaticales et de vocabulaire. La remise en cause de savoirs se fait plus facilement si l'on sort de la langue codée de la science. Nous reviendrons plus loin sur cette dimension cognitive de la langue après avoir envisagé d'autres situations où mon type de « bilinguisme » ne suffit pas.

Dès que je sors de mon champ de compétence et de curiosité scientifique, j'ai beaucoup plus de mal à m'exprimer en anglais. Le problème ne consiste pas tant à traduire un vocabulaire technique dont je disposerais en français - je sais me servir de dictionnaires spécialisés monolingues ou bilingues (le Random House Dictionary, unabridged edition, est mon préféré) - qu'à utiliser une expression plus générique. Hors de mon univers spécialisé, je dois recourir à une langue plus « naturelle », par exemple, lorsqu'il s'agit de décrire un montage expérimental un peu complexe ou une photo de phénomène. Les langues naturelles ont évidemment toute la plasticité nécessaire pour suppléer les langages codés des spécialistes. Dans le cas d'un dispositif expérimental à décrire en anglais je suis un peu "coincé " dans la mesure où je ne suis ni anglophone natif, ni technicien de laboratoire. Heureusement, nous sommes aidés par d'autres outils de description très robustes tels que formules, diagrammes, schémas, photos, etc. L'utilisation de phrases courtes renvoyant, autant que possible, aux éléments iconographiques fait le reste.

Il est intéressant de noter que, même si c'est à un niveau bien moindre, je ressens également ce différentiel d'aisance en français entre l'écriture d'un article de physique et celle d'un texte sur un autre sujet scientifique ou technique ${ }^{3}$. On voit là certainement le signe que l'habileté à exprimer des résultats issus de la manipulation des concepts, des objets propres à son domaine est d'une autre nature que l'aisance à s'exprimer sur des sujets moins objectivés. Il n'y a pas la même implication de la personne, de sa culture, de sa psychologie.

11 Ces langues naturelles sont des langues porteuses de culture. À ce titre, l'anglais ne diffère pas des autres langues, mais il souffre d'une fâcheuse confusion entre sa version naturelle, originale, celle parlée par les anglophones, et sa version franche (au sens de lingua franca) scientifique. Ces deux versions, bien qu'elles partagent beaucoup de traits ce qui donne encore un net avantage aux anglophones dans la communication internationale - méritent d'être distinguées, en particulier au moment de l'apprentissage scolaire. Il convient de savoir ce qu'on apprend, à quel usage cet anglais est destiné. Les élèves scientifiques de l'École Normale Supérieure ont à la fois des cours d'anglais scientifique adaptés dans leurs cursus de magistère respectifs et suivent souvent des cours de langues (dont l'anglais « culturel ») aux côtés de leurs camarades littéraires.

\section{D'autres usages de la langue maternelle en sciences}

12 Je viens de décrire successivement mes compétences, puis mes difficultés dans la communication écrite ou orale en anglais scientifique. Il s'agissait de circonstances 
requérant une négociation linguistique, c'est-à-dire des situations où la présence d'interlocuteurs ou de destinataires du discours appelle, en général - nous verrons plus loin que d'autres solutions existent - l'usage de cette langue tierce (pour beaucoup d'entre nous) qu'est l'anglais. Mais toutes les occasions de s'exprimer ne se résument pas à la communication avec autrui. Il existe également tout un travail de formulation pour soi-même. Je retiendrai trois exemples.

13 À un niveau élémentaire, dans le simple calcul mental, au long de mon activité d'enseignement et de recherche à l'étranger, il est clair que je n'ai pensé mes chiffres et n'ai calculé qu'en français.

14 Regardons, également, un cahier de notes ou de manipulation. Il s'agit d'un document à usage personnel, même s'il rend compte souvent d'un travail fait en équipe et doit pouvoir être consulté par d'autres, ne serait-ce que dans des recherches d'antériorité de découverte. On constate qu'en général ce cahier est tenu dans la langue de culture maternelle quel que soit le pays où l'on travaille. Les notes écrites contiennent des descriptions de phénomènes ou d'idées en cours de découverte. L'expression y est libre. Cette appropriation de nouveaux savoirs nécessite une agilité et une disponibilité mentale que nous n'avons que dans notre propre langue maternelle. Il nous faut utiliser toutes les ressources culturelles dont nous disposons pour favoriser la naissance de ce qui n'est pas encore.

En conférence, la prise de notes subit deux influences linguistiques : celle du conférencier et celle de l'auditeur qui prélève des morceaux du discours. Il serait intéressant de voir quels sont les choix de langue et même comment alternent ces langues dans les relevés. Dans un exposé en anglais dont je maîtrise le contenu, j'écrirai en français un résumé ou mon interprétation de ce que j'entends. Je noterai cependant en anglais des phrases d'un discours trop rapide, mal compris ou, au contraire des phrases particulièrement cruciales dans leur expression exacte. Je dois à l'influence de Pierre-Gilles de Gennes, lorsque je faisais ma thèse, de ne pas écrire exactement ce que j'entends afin de forcer une appropriation personnelle et de ne pas lire exactement le texte d'un exposé oral. Une façon efficace de se détacher de ses notes écrites consiste à les rédiger dans une autre langue.

\section{L'expression des idées en langue maternelle}

\section{Nécessité cognitive}

Cette phase antérieure à la communication, qui correspond à une formulation, une formalisation de ses propres idées se fait naturellement dans la langue de son for intérieur, dans sa langue intime. Cela est particulièrement flagrant pour une vraie découverte, c'est-à-dire pour une idée ayant un caractère suffisant d'originalité. "Une idée, mais c'est si rare!» a écrit Albert Einstein. C'est rare, mais c'est aussi fragile ; et pour suivre le fil conducteur d'une nouvelle idée, qu'elle se soit imposée par une révélation fulgurante ou par le jeu du hasard que l'on qualifie aujourd'hui de sérendipité, il nous faut mobiliser l'ensemble de nos facultés cognitives. Somme d'inné et d'acquis, l'idée nouvelle n'accepte pas aisément la réduction d'une expression dans la langue véhiculaire, celle qui est si bien adaptée à l'existant, mais qu'il faut bousculer pour lui faire intégrer le nouveau. 
17 Et pourtant, l'idée ne devient elle-même que dans son expression. Et son expression première est celle que lui offre la langue. Elle peut apparaître, bien sûr, sous forme d'une équation ou d'une courbe. Mais, j'ai le sentiment que ces formes ont d'abord été pensées dans la signification que leur permettait une langue naturelle. Des formules aussi compactes et pleines de sens pour un physicien que $\mathrm{E}=\mathrm{mc}^{2}$ ou $\mathrm{H}^{\mathrm{y}}=\mathrm{E}^{\mathrm{y}}$ sont parties pour Einstein et Schrödinger de l'image classique, exprimable par des mots, de l'énergie et de sa conservation ou de la propagation d'une onde matérielle. Il semble peu probable que de telles équations ne soient pas apparues très tôt au travers d'une formulation linguistique, en allemand en l'occurrence. Cette langue naturelle porte un sens qui nous permet toujours de nous approprier l'abstrait de telles formules, nous permet de lui donner un sens par le secours de l'analogie et de les rattacher à du connu plus élémentaire ou plus proche.

\section{Plasticité du signifiant}

Mais cette langue possède une autre caractéristique : à partir d'une formulation, elle autorise des glissements de sens, grâce aux connotations, à toutes les charges culturelles, émotionnelles qui accompagnent les mots, les séquences syntaxiques de cette formulation. Prenons par exemple, les mots «chaos», "attracteur étrange» ou " catastrophe » qui accompagnent des champs de découvertes récentes importantes et bien précises. Le choix même de ces mots forts a permis une récupération floue et abusive audelà de leur champ propre de définition scientifique. On peut d'ailleurs se demander quel rôle le sens commun, familier, de ces mots a joué dans l'engouement (pas seulement médiatique ou grand public) pour ce domaine. Et au-delà de cet engouement, peut-on vraiment exclure que la créativité scientifique dans ce domaine doive quelque chose à l'imaginaire des hommes de sciences jouant avec de tels mots? En physique des particules élémentaires (le CERN est en zone francophone!) ${ }^{4}$, une certaine fantaisie lexicale a permis de catégoriser les particules élémentaires selon leur "charme ", "beauté ", "couleur ", mots d'ailleurs voisins en français et en anglais. Et si leur propre expérience de tels attributs ne peut aider à des découvertes nouvelles au moins concédera-t-on que les physiciens avaient un certain besoin d'accrocher leur description scientifique à des images plus immédiates et courantes. Que l'on songe également à la motivation du prix Nobel Murray Gell Mann, qui trouva dans le roman Finnegan's Wake de James Joyce le nom à donner à la particule élémentaire dont il venait de proposer l'existence après de très savants et très abstraits calculs (le quark). La stabilisation d'une notion, son incarnation dans un registre lexical indépendant, n'est pas seulement anecdotique ou historique. Elle témoigne du besoin de l'homme de science de maintenir le contact entre son discours technique et ses références culturelles. Cela aide la mémoire. Pourrait-on envisager une science dont la terminologie serait faite de mots, simples chaînes de caractères, aléatoirement distribués sur les entités manipulées par cette science?

19 Il nous faut donc accepter les mots et la langue, dans leur force de création comme dans les déviations qu'ils autorisent, et reconnaître, à côté, les vertus d'un anglais de spécialité qui s'affirme par la précision simplificatrice qu'il autorise. La leçon inaugurale de Pierre Gilles de Gennes au Collège de France portait sur l'ambivalence des archétypes qui fournissent à la fois les représentations analogiques nécessaires, mais, dans le même temps, enferment celui qui les utilise. Ce n'est sans doute pas neutre et innocent qu'il ait 
dans un article de La Recherche inventé l'expression « la fourmi dans un labyrinthe » pour décrire la marche au hasard dans un réseau mal interconnecté5. Les développements, en physique du désordre, de ce concept nouveau qu'il introduisait dans le même temps, ont été considérables. La formule associe deux mots courants, fourmi et labyrinthe, et les images fortes qu'ils véhiculent: celle du fourmillement, de l'essaim, et celle du labyrinthe, d'un chemin mal et multiplement connecté. Il s'agit en plus d'un animal, donc d'un être doué d'intentions (au moins d'un instinct) et d'une construction artificielle destinée à un certain usage. Dans la description d'un phénomène aléatoire, on a donc introduit une sorte de téléonomie qui oriente certainement le chercheur dans une certaine façon d'appréhender le problème. Dans les travaux qui ont suivi, les chercheurs ont utilisé des fourmis douées d'odorat, de mémoire, aveugles...

Le mot, l'expression, créés accompagnent ainsi et souvent la découverte, et l'appropriation dans une langue familière est une démarche essentielle. J'ai moi-même contribué à fédérer une communauté nationale autour de l'acronyme « MIAM » (milieux aléatoires macroscopiques) puis autour de "la physique du sac de billes » qui donnait une bannière à la science des milieux granulaires. Une activité importante en France s'est développée depuis une vingtaine d'années sous ces deux étiquettes, ce qui a permis que le reste de la communauté internationale reconnaisse le champ sous sa dénomination française en reconnaissant au même temps une antériorité de recherche. Tant mieux pour les scientifiques français qui se meuvent ainsi dans un milieu plus familier que si leur discipline s'appelait d'un nom japonais.

\section{Effets d'image et prise de pouvoir}

L'effort de terminologie et de prise de possession par la langue touche également à un domaine plus sociologique, économique et politique, externe à la science. Il s'agit pour différents pays ou communautés linguistiques de faire valoir une domination ou, au moins, une présence. Des stratégies proches du marketing se manifestent: le mathématicien Benoît Mandelbrot, Français ayant fait carrière aux États-Unis, à l'instar de George Eastman créant le mot Kodak pour l'appareil de prises de vue qu'il voulait commercialiser, a cherché un mot qui se dise dans des langues diverses, sans avoir à être traduit, pour dénommer une famille d'objets mathématiques. À la description de géométries présentant des échelles multiples de tailles et semblables à elles-mêmes, aboutissement d'un demi-siècle de travaux de mathématiciens de la théorie de la mesure, il associe le mot fractal, de racine latine. Cette appellation authentifie l'origine de la découverte plus sûrement qu'un brevet. De plus, le mot est neuf et permet d'éviter les dérapages de sens.

Il n'est pas besoin d'être le « titulaire » d'une invention pour s'intéresser à l'expression en français d'une notion. Les articles de revue et les traductions d'ouvrage en français destinés à stabiliser des connaissances, ce dont nous parlions en début d'article, nous offrent souvent l'occasion de faire ce travail de terminologie ou de néologie. Malheureusement, les séminaires scientifiques en français se contentent souvent de l'utilisation du mot anglais. Ainsi un physicien français continuera à parler de cluster, le mot amas lui semblant trop banalisé.

Le manque d'imagination de scientifiques écrivant en français dans des articles de revue est parfois désolant. Il semble que le rôle de la langue ne soit pas perçu comme il convient. Un scientifique de bon aloi est souvent sommé de se ranger parmi les 
défenseurs de la France (donc préoccupés de faire barrage à l'anglais) ou parmi les défenseurs de la Science (donc de l'anglais). Cette dichotomie fausse un débat plus subtil qui pourrait être mené tant avec les linguistes (traducteurs, rédacteurs, terminologues, et autres langagiers) qu'avec les pouvoirs publics ou les éditeurs ou tout autre acteur de la diffusion du savoir scientifique.

\section{Vertus de la diversité linguistique}

La nécessité que nous ressentons de nous exprimer dans notre langue maternelle au moment où nous mettons en forme des savoirs, mais aussi lorsque nous les partageons avec un plus grand nombre parlant la même langue maternelle que nous, dans des actions relevant de la formation ou de la culture scientifique grand public, ne s'oppose pas, bien sûr, à l'utilisation d'une langue véhiculaire commune, l'anglais de spécialité lorsque la cible est internationale. Mais elle suggère un plus grand respect de la diversité d'expression. Ce que j'ai décrit de mon fonctionnement, de mon rapport à l'anglais, me semble transposable à tous les locuteurs ayant une langue maternelle autre que l'anglais. Cette prise de conscience m'engage à avoir un comportement linguistique qui minimise les frustrations dues au recours à une langue code tierce. Il s'agit donc de développer une approche plus interculturelle toutes les fois que cela est possible comme alternative à l'utilisation exclusive d'un code pivot. Cela sera d'autant plus pratiqué que la connaissance de langues étrangères se développera chez chacun. Il s'agit d'augmenter la compétence en langues dans la population et, en l'occurrence, chez les scientifiques. L'expérience que nous avons des concours d'entrée à l'École Normale Supérieure nous montre qu'une fraction très importante des élèves (plus de $30 \%$ ) pratique l'allemand en première langue, ce qui est très supérieur à la moyenne nationale de $10 \%$. Cela est bien sûr, lié à la sélection de fait qu'opère cette langue dès la classe de $6^{e}$, comme le faisait le latin antérieurement. Par ailleurs, nous avons deux langues étrangères obligatoires au concours sciences, et j'ai donné le même poids à la seconde langue qu'à la première. J'ai été aidé au long de ce projet par Antoine Culioli. Le résultat est spectaculaire : une langue autre que l'anglais ou l'allemand sera choisie par $40 \%$ des candidats comme seconde langue étrangère (parmi ces candidats, un tiers choisit le latin ou le grec ancien). Dans le concours de l'ENS-Lyon, dont l'origine de recrutement est la même que l'ENS-Ulm mais qui a une seule langue vivante au concours, comme la plupart des concours sciences des grandes écoles, le choix de la langue étrangère unique reste limité à l'anglais et l'allemand. Cela, à soi seul, montre qu'il faut encourager la pratique et l'entretien d'au moins deux langues étrangères, l'une étant alors inévitablement l'anglais ${ }^{6}$.

\section{Le dialogue polyglotte}

De façon pratique, il faut considérer les possibilités de communication entre deux locuteurs allophones. Il existe une solution que l'on appelle parfois le dialogue polyglotte. Tout en sachant qu'elle aura une portée quantitative limitée, il importe de mesurer sa valeur au niveau humain et de l'évaluer comme modèle possible.

Pour illustrer le sens et la faisabilité de cette solution, je voudrais revenir sur le témoignage personnel du physicien, peu doué pour les langues vivantes, mais motivé par les contacts humains et scientifiques authentiques. J'ai appris au long de ma carrière 
suffisamment d'italien, d'espagnol pour pouvoir écouter une conférence ou une conversation dans l'une de ces langues, d'allemand et de russe pour pouvoir approcher des textes ou des échanges oraux simples. Cela change-t-il quelque chose dans les rencontres qui peuvent être faites dans un des pays où se pratiquent ces langues ? À coup sûr, oui. Au niveau de la rencontre toute simple ${ }^{7}$. Mais aussi au niveau de l'élaboration commune d'idées nouvelles où il importe que chacun puisse s'exprimer le plus naturellement possible dans la langue la plus familière tout en s'efforçant de rencontrer l'autre dans son expression familière. Depuis plusieurs années, je participe à des échanges réguliers avec divers pays d'Amérique du Sud. Nous avons pris pour parti, et pour habitude de n'utiliser l'anglais que lorsqu'une tierce partie non familière avec l'espagnol présente, ou lors des rencontres internationales où l'anglais est resté de règle ${ }^{8}$. Il y a derrière ce choix, au-delà même d'une attitude politique, une optimisation à rechercher lorsque trois langues sont en jeu dans une rencontre. Ce qui va suivre est un essai de formalisation des situations de communication polyglotte et des effets sur la qualité d'expression et de réception des messages.

\section{Essai}

Mener une conversation en langue non maternelle demande un certain effort et coûte également quelques pertes dans l'émission et dans la réception des messages. Voyons l'économie de ces efforts et de ces pertes selon les choix linguistiques effectués à partir d'un modèle très schématique. Dès lors qu'un échange oral met en jeu des locuteurs de langues maternelles différentes, un coût et des pertes se produiront.

28 La réponse la plus naturelle consiste à chercher une langue unique, commune pour l'ensemble des locuteurs et pour toute la durée de l'échange. Cela a pour conséquence qu'il y a toujours au moins un des groupes linguistiques en présence qui doit parler et comprendre une langue étrangère. Il se peut même que tous les groupes soient dans cette situation si on recourt à une langue tierce. C'est alors généralement l'anglais qui est choisi car il est aujourd'hui automatiquement identifié comme le plus probable dénominateur commun. L'anglais de spécialité est en effet, le plus grand commun dénominateur de communication de l'ensemble de la communauté scientifique.

Le dialogue polyglotte est une solution de rechange par rapport à cette tendance. Il consiste en ce que chacun parle sa langue et comprenne celle de l'autre. Plusieurs langues seront donc présentes dans l'échange.

Statistiquement, cette solution semble, à première vue, peu applicable et peu intéressante puisqu'il faut que tous les interlocuteurs en présence connaissent toutes les langues maternelles des autres. Il sera, en outre, difficile de déterminer pour chaque conversation si c'est le cas. On pourrait juger que, tant qu'à être contraint d'utiliser une langue étrangère, autant choisir l'anglais qui a toutes les chances d'être connu de tous. C'est sans compter sur plusieurs faits :

31 - une majorité de conversations entre locuteurs de langues différentes ne met en présence que deux langues, ce qui augmente considérablement la probabilité d'avoir une langue commune ;

32 - il existe des degrés de proximité entre les langues en présence, qui tiennent à leur voisinage géographique ou à une histoire passée et qui favorisent d'autres choix de langue véhiculaire ; 

(2)

\section{(1) Considérations qualitatives} pourra s'exprimer plus finement dans sa propre langue, utiliser des expressions plus riches, établir des reformulations explicatives, etc. jusqu'à la limite de compréhension de son interlocuteur. Le différentiel entre le niveau productif et réceptif des auditeurs est ainsi exploité.

Bien sûr, en dessous d'un certain seuil de compétence réceptive des auditeurs, il vaut mieux passer par une langue tierce. Mais, il ne faut pas perdre l'idée que, si les compétences des interlocuteurs sont égales dans cette langue tierce, l'émetteur n'atteindra jamais le niveau de finesse que les récepteurs auraient encore pu interpréter.

\section{... de formalisation}

On peut essayer de formaliser cela en disant que la limite supérieure de qualité d'un message transmis peut se définir comme un plus grand diviseur commun entre la qualité du message émis et la qualité du message reçu.

pur laquelle nous nous fondons est qu'il est plus facile d'obtenir une meilleure qualité réceptive qu'une bonne qualité d'émission. Les interlocuteurs doivent alors chercher à optimiser cette limite en jouant sur la phase d'émission.

Pour déterminer où se trouve cette limite, on compare le niveau de compréhension du récepteur au niveau d'expression de l'émetteur. Si le message est dans la langue de l'un des deux, le résultat de la comparaison est évident. S'il est dans une langue tierce, la réponse dépendra des compétences particulières des individus en jeu. Mais à compétences égales, la réponse sera, justement, que le niveau du récepteur est un cran au-dessus de celui de l'émetteur.

41 Comment optimiser la qualité d'une conversation dans son ensemble et non seulement celui d'un message orienté ? Il s'agit de comparer la qualité des flux de messages dans chaque sens. Pour simplifier, on supposera des niveaux de compétence symétriques entre les interlocuteurs et des compétences identiques pour toutes les langues en jeu.

Le locuteur $A$ a pour langue maternelle première $a$ et connait les langues $b$ et $c$, alors que le locuteur B a pour langue maternelle le b et connaît les langues a et $c$.

\section{Notons le niveau d'expression de A en langue $\mathrm{c}$ :}

$$
\exp (A, c)
$$

et le niveau de compréhension de $A$ en langue $c$ :

$\operatorname{comp}(A, c)$

On peut établir une hiérarchie à 3 niveaux (croissants) :

$$
\begin{aligned}
& 1=\exp (\mathrm{A}, \mathrm{b})=\exp (\mathrm{A}, \mathrm{c})=\exp (\mathrm{B}, \mathrm{a})=\exp (\mathrm{B}, \mathrm{c}) \\
& 2=\operatorname{comp}(\mathrm{A}, \mathrm{b})=\operatorname{comp}(\mathrm{A}, \mathrm{c})=\operatorname{comp}(\mathrm{B}, \mathrm{a})=\operatorname{comp}(\mathrm{B}, \mathrm{C}) \\
& 3=\exp (\mathrm{A}, \mathrm{a})=\exp (\mathrm{B}, \mathrm{b})=\operatorname{comp}(\mathrm{A}, \mathrm{a})=\operatorname{comp}(\mathrm{B}, \mathrm{b})
\end{aligned}
$$



que je dois fournir pour parler une langue donnée est-il supérieur à celui que doit fournir mon interlocuteur pour comprendre cette même langue? Les résultats iraient dans le même sens. L'effort maximal étant fourni lors du recours à une langue tierce. Qu'il s'agisse de qualité du message ou d'économie personnelle, il y aurait toujours avantage au dialogue polyglotte. En fait, une étude pourrait être menée sur l'évaluation des niveaux de compétences en production et en réception dans l'optique du dialogue polyglotte. On pourrait alors sortir de ce cas d'école où les langues sont parlées au même niveau en tant que langues secondes par les deux interlocuteurs. Il faudrait, en outre, savoir à quel rapport de niveaux entre les compétences en langue tierce et dans la langue de l'autre se joue l'intérêt de cette solution de communication.

Qu quilen soit, nous restons convaincus que la qualté des rapports humains gagnera toujours à travers un plurilinguisme où apparaît une marque de respect et de reconnaissance fondamentale, lorsque l'on accepte d'entendre la langue de l'autre.

\section{(2) Considérations quantitatives}

D'autre part, le nombre de personnes "passivement" polyglottes augmente. Cette méthode a donc, pour avantage d'augmenter considérablement les chances de dénominateur commun autre que l'anglais.

Un travail statistique serait à faire sur la répartition des compétences et sur leur niveau afin de mesurer la validité de la solution polyglotte. C'est-à-dire de savoir quelle extension ce mode d'échange pourrait avoir dans la communication entre les citoyens, que ce soit dans leurs activités spécialisées ou dans leurs rapports plus personnels.

Mais, il ne suffit pas d'avoir fait des calculs d'optimisation qualitative, et d'avoir mesuré la faisabilité de la solution polyglotte sur une population pour que la population en question l'adopte en bloc. Des freins venant de la culture, des mentalités, des croyances, des habitudes s'y opposent ${ }^{9}$. 


\section{Conclusion}

56

Lidée scientifique n'existe vraiment que lorsqu'elle trouve son expression langagière C'est alors que s'effectue le passage de la réalité perçue à la phase de l'interprétation. Un phénomène n'acquiert son statut de phénomène qu'à partir du moment où il a reçu une définition, où on le distingue comme tel au sein de la substance compacte mais diffuse qu'est la réalité, l'espace spatio-temporel de notre existence. C'est précisément ce passage du continu au discret que permet le langage. Et toutes les langues n'ont pas les mêmes grains, les mêmes outils, pour opérer ce passage. Bien sûr, toutes les langues peuvent reproduire un découpage donné de la réalité, mais avant que ce découpage ne soit admis comme intéressant, comme pertinent, beaucoup d'autres ont été essayés, bénéficiant des matériaux de base, des mécanismes familiers que la langue offre à la pensée en première approche.

La mission des enseignants réunis dans ce colloque du GERAS est délicate. Il importe aujourd'hui que les étudiants qu'ils forment à l'anglais de spécialité soient conscients de l'importance de l'apprentissage technique de l'anglais sous les formes diverses dont les actes du colloque se font l'écho. Tant sur le plan de l'écrit que de l'oral, les jeunes cadres et universitaires français souffrent souvent de la comparaison, en particulier avec les pays du nord de l'Europe, dans l'expression d'une langue scientifique et technique permettant des échanges internationaux tous azimuts et, aujourd'hui, indispensables. Clairement, la formation classique en anglais, si elle est précieuse pour l'enrichissement de nos échanges culturels avec les pays anglophones, n'a que peu à voir avec le «sabir international» que le scientifique pratique habituellement. Mais il importe que ce nécessaire apprentissage ne se fasse pas au détriment de l'apprentissage linguistique culturel ni de la curiosité pour d'autres langues étrangères (de langues de culture ou de langues véhiculaires secondaires). Cela s'applique, en particulier, pour les francophones, par rapport à l'apprentissage des autres langues latines. Construire les modalités d'un plurilinguisme, qui respecte la diversité des approches sans pour autant s'opposer à l'efficacité de la communication spécialisée demande, en particulier, qu'une articulation harmonieuse soit trouvée avec les usages incontournables de l'anglais de spécialité10.

\section{ANNEXES}

\section{AN EXPERIMENT REGARDING THE WAVE FUNCTION OF SUPERFLUID HELIUM}

M. Chester, E. Guyon and B.K. Jones University of California, Los Angeles, California, U.S.A. (Received 10 July1967 by P. G. de Gennes) (In revised form 14 August 1967)

Texte reproduit avec l'aimable permission de Solid State Communications, Vol. 5, pp. 807808, Oxford : Pergamon Press Ltd., 1967.

Using as a thermal detector a granular carbon film with an area of about $25 \mathrm{~m}$ on a side we have looked for a possible diffraction pattern caused by liquid helium II flowing 
through a wire grating. At a detection distance of about $1 / 4$ in. from the grating we observed no effect to within about 10 microdegrees.

Nous avons étudié la possibilité de diffracter un courant d'helium II par un réseau fait de fils parallèles. La modulation de température mesurée à une distance de $1 \mathrm{~cm}$ du réseau à partir d'une microsonde de carbone est nulle à 10 microdegrés près.

\begin{tabular}{|c|c|}
\hline $\begin{array}{l}\text { Helium II is a liquid quite strange. } \\
\text { It flows without viscosity. } \\
\text { It goes through holes and powder packed tubes of } \\
\text { exceedingly low porosity. }\end{array}$ & $\begin{array}{l}\text { We made such a grating with milli-inch } \\
\text { wires } \\
\text { and milli-inch gaps in between. } \\
\text { The flow was controlled by the fountain } \\
\text { effect, } \\
\text { And a four-micron wave was the mean. }\end{array}$ \\
\hline $\begin{array}{l}\text { But strangest of all and astounding indeed } \\
\text { is this quantum mechanical attribute. } \\
\text { Helium liquid possesses a phase } \\
\text { as well as an amplitude absolute. }{ }^{1}\end{array}$ & $\begin{array}{l}\text { The speed of the flow was less than } \mathrm{V}_{\mathrm{c}} \\
\text { when past the grating directed. } \\
\text { 'Fine pores at one end served to block } \\
\text { normal flow and } \\
\text { our signal was lock-in detected. }\end{array}$ \\
\hline $\begin{array}{l}\text { The amplitude comes from } \rho_{8} \text { over } \rho \text {, } \\
\text { and the wavelength's de Broglie's relation. }{ }^{2} \\
\text { These describe a coherent, potential type flow } \\
\text { and give quanta of flow circulation. }{ }^{3}\end{array}$ & $\begin{array}{l}\text { On a two-fluid model this should have } \\
\text { produced } \\
\text { at the proper Bragg angle condition, } \\
\text { a temperature drop of rather large size } \\
\text { if } \Psi \text { obeys superposition. }\end{array}$ \\
\hline $\begin{array}{l}\text { Now everyone knows that the phase of a wave } \\
\text { will surely give rise to diffraction, } \\
\text { If a regular grating is put in the way } \\
\text { and the wave suffers some interaction. }\end{array}$ & $\begin{array}{l}\text { The equation for } \Psi \text { has non-linear terms } \\
2 \\
\text { these violate superposition. } \\
\text { But how much was not known, nor over } \\
\text { what range, } \\
\text { nor the time constant clear in addition. }\end{array}$ \\
\hline & $\begin{array}{l}\text { Our thermal detector }{ }^{5} \text { was able to see } \\
10 \text { microdegrees we are positive. } \\
\text { Its size was quite small, the angle was } \\
\text { right; } \\
\text { Our findings, however, were negative. }\end{array}$ \\
\hline
\end{tabular}

${ }^{1}$ ANDERSON P.W., Rev. Mod. Phys. 38, 298 (1966).

2 PITAEVSKII L. P., Soviet Physics - JETP 8, 282 (1959). More recent accounts indicate that the amplitude comes from $\mathrm{N}_{\mathrm{o}}$, the number of atorns in the condensed state, rather than $\mathrm{r}$. (see Ref. 1), but this makes little difference for this experiment. 
${ }^{3}$ VINEN W. F., Proc. Roy. Soc. (London) A260 218 (1961).

${ }^{4}$ The critical velocity $-\mathrm{v}_{\mathrm{c}}$ - is discussed, for example, in LONDON F., Superfluids, Vol. II, p.147, Wiley, New York (1954)

${ }^{5}$ CANNON W. C. and CHESTER M., Rev. Sci. Instrum. 38, 318 (1967).

\section{NOTES}

1. Chester, M., E. Guyon and B. K. Jones1967. Solid state communications 5: 807. Voir reproduction $\mathrm{du}$ poème en question en annexe.

2. Invité à l'Université de Montréal pour faire la conférence inaugurale de la bilingue association des physiciens canadiens, je me suis fait expliquer préalablement que soit je faisais la conférence en français et qu'elle ne serait pas comprise par les 3/4 des participants, soit je la faisais en anglais et cela serait considéré à Montréal comme injurieux. J'ai finalement décidé de la faire en français en l'illustrant suffisamment (le sujet, la physique des cristaux liquides s'y prêtait) et en utilisant de nombreux transparents reproduisant ce que je disais, écrits non en anglais mais en français. La connaissance du français administratif écrit est telle au Canada que de nombreux collègues purement anglophones m'ont dit que c'était la première conférence en français qu'ils comprenaient totalement.

3. La lecture même de certains articles en français, comme ceux de cette superbe Revue qu'édite le Musée des Arts et Métiers, n'est pas sans me poser des problèmes de compréhension des mots techniques.

4. Hmm ! J'ai rencontré récemment un physicien américain, prix Nobel de physique, travaillant au CERN depuis plusieurs années et qui a même acquis une maison dans les environs, totalement incapable de s'exprimer en français. Rassurez-vous! On ne parle qu'anglais ou presque entre savants au CERN.

5. L'expression est devenue internationale... et je sais dire fourmi dans une demi-douzaine de langues étrangères !

6. Dans son dernier ouvrage, l'enfant aux deux langues, Claude Hagège suggère même qu'il serait souhaitable de diversifier le choix de la première langue apprise à l'école en excluant l'anglais qui, dans tous les cas, sera appris et pratiqué ultérieurement. Une telle proposition provocante avait été faite il y a plus de vingt ans par Bernard Cassen dans le numéro 1 du Monde de l'éducation en 1974. Il faut cependant faire attention à ce que de telles solutions, irrecevables par notre société, ne soient pas l'alibi pour introduire d'autres mesures qui, sans ce garde fou, constituent précisément un danger pour la diversité linguistique.

7. Le décalage entre le caractère universel des échanges scientifiques et les échanges culturels qui accompagnent heureusement le travail de recherche international peut être illustré dans un article écrit avec un collègue japonais. Dans une lettre écrite de Sendai, qui ne comportait qu'un ensemble de formules liées par des thus; again; It results... la lettre se terminait brutalement en français par la phrase «Oh, qu'il est doux d'entendre le chant du koto dans les jardins calmes et frais». J'imagine que mon correspondant me décrivait les conditions dans lesquelles il avait écrit sa part de la rédaction de l'article commun...

8. Dans des rencontres bisannuelles de physique au Chili auxquelles je participe depuis 8 ans, et qui associent largement une communauté de physiciens français aux physiciens chiliens et plus généralement sud-américains avec d'autres invités principalement des USA et d'Allemagne, j'ai $\mathrm{pu}$ constater une évolution très nette d'une rencontre à l'autre. Ces rencontres sont véritablement devenues trilingues. L'anglais est majoritairement utilisé pour les communications orales, avec un plus faible pourcentage de communications en français et en espagnol. Mais les 
débats et les rencontres autour de ces exposés se pratiquent très librement et de façon équilibrée dans les trois langues.

9. Cf. les travaux de Roland Posner, qui affirment que le dialoque polyglotte ne pourra s'instaurer en Europe de l'Ouest que si on change les principes de politesse, d'efficacité de la communication, de méthodes d'enseignement scolaire qui lui soient propres et qui privilégient l'homogénéité linguistique. (Roland Posner, "Gesellschaft, Zivilisation und Mentalität: Vorüberlegungen zu einer Sprachpolitik für Europa», Saarbrücken : Breitenbach, 1990).

10. Pour une réflexion au niveau européen, on se reportera aux travaux du colloque «Construire le pluralisme linguistique : quelles initiatives pour l'Europe ?", tenu à Besançon les 19 et 20 juin 1995. Les actes peuvent en être obtenus auprès de l'Observatoire linguistique, 3 faubourg de Tarragnoz, 25 000 Besançon.

\section{RÉSUMÉS}

L'article est une version remaniée de la conférence d'ouverture prononcée par le professeur E. Guyon lors du 17e Colloque du GERAS, Aix-en-Provence, mars 1996. Il constitue le précieux témoignage de première main d'un éminent scientifique français, et doit à ce titre être considéré comme une base fiable de réflexion pour tous les anglicistes qu'intéresse la formation des spécialistes d'autres disciplines à l'utilisation de l'anglais pour les besoins de leur profession. Le bilinguisme du scientifique est ici présenté comme l'aptitude à manier un code minimal, présenté comme l'outil le plus adéquat de la communication entre chercheurs internationaux à propos de «l'objet réputé universel, la Science». En corollaire se manifeste un vibrant plaidoyer pour le nécessaire « dialogue polyglotte » complémentaire. La relation dynamique entre l'une et l'autre utilisation de la langue fait l'objet d'une tentative de formalisation.

This article is a revised version of the opening lecture given by Professor E. Guyon at the 17th GERAS Conference in Aix-en-Provence, March 1996. It is to be read as a rare piece of first-hand testimony by one prominent French physicist: as such, it will have to be taken into consideration by ESP researchers and teachers alike, involved in the training of students and colleagues in the use of English for the needs of their professional activity. The scientist's bilingualism is here defined as the aptitude to use a minimal code of communication, the most efficient tool in international scientific exchanges about that so-called "universal object, Science". As a parallel development, the authors also advocate "multilingual dialogue" as a necessary complement for human and cultural communication. A formalisation of the dynamic relation between these two ways of using language is attempted.

\section{INDEX}

Mots-clés : anglais scientifique, dialogue polyglotte, publication scientifique

Keywords : multilingual dialogue, scientific English, scientific publication 


\section{AUTEURS}

\section{ÉTIENNE GUYON}

Physicien, Directeur de l'École normale Supérieure.guyon@pmmh.espci.fr

\section{ANNE GUYON}

DISTNB, Ministère de l'Éducation nationale, de l'Enseignement supérieur et de la Recherche 